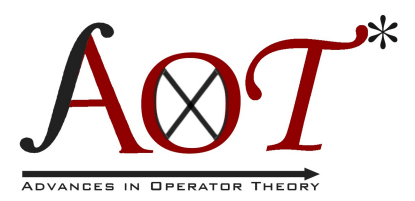

Adv. Oper. Theory 4 (2019), no. 1, 99-112

https://doi.org/10.15352/aot.1712-1275

ISSN: 2538-225X (electronic)

https://projecteuclid.org/aot

\title{
CONVOLUTION DOMINATED OPERATORS ON COMPACT EXTENSIONS OF ABELIAN GROUPS
}

\author{
GERO FENDLER ${ }^{1 *}$ and MICHAEL LEINERT ${ }^{2}$ \\ Communicated by L. Turowska
}

\begin{abstract}
If $G$ is a locally compact group, $C D(G)$ the algebra of convolution dominated operators on $L^{2}(G)$, then an important question is: Is $\mathbb{C} 1+C D(G)$ (or $C D(G)$ if $G$ is discrete) inverse-closed in the algebra of bounded operators on $L^{2}(G)$ ?

In this note we answer this question in the affirmative, provided $G$ is such that one of the following properties is satisfied.

(1) There is a discrete, rigidly symmetric, and amenable subgroup $H \subset G$ and a (measurable) relatively compact neighbourhood of the identity $U$, invariant under conjugation by elements of $H$, such that $\{h U: h \in H\}$ is a partition of $G$.

(2) The commutator subgroup of $G$ is relatively compact. (If $G$ is connected, this just means that $G$ is an IN group.)

All known examples where $C D(G)$ is inverse-closed in $B\left(L^{2}(G)\right)$ are covered by this.
\end{abstract}

\section{INTRODUCTION}

For an operator on Hilbert space with an additional property, often this property is not preserved under inversion. So there is an interest in situations where this does not happen. For example, consider on $l^{2}(\mathbb{Z})$ an operator as a twosided infinite matrix; then it might have a certain off-diagonal decay; that is, its entries $a_{i, j}$ decay as $k=|i-j|$ becomes large. A condition of summability

Copyright 2019 by the Tusi Mathematical Research Group.

Date: Received: Dec. 16, 2017; Accepted: Apr. 3, 2018.

* Corresponding author .

2010 Mathematics Subject Classification. Primary 47B35; Secondary 43A20.

Key words and phrases. Convolution dominated operator, generalised $L^{1}$-algebra, symmetric locally compact group. 
like $\sum_{k} \sup \left\{\left|a_{i, j}\right|:|i-j|=k\right\}<\infty$ is an example. This type of condition is preserved under inversion. Note these operators $A$ are characterised by the condition that there exists an $\alpha \in l^{1}(\mathbb{Z})$ dominating the operator in the sense that $|A(\xi)(l)| \leq \sum_{k} \alpha(k)|\xi(l-k)|$, for example, $\alpha(k)=\sup \left\{\left|a_{i, j}\right|:|i-j|=|k|\right\}$. With canonical operations the set of these operators is a Banach $*$-algebra. To see that the set is closed under multiplication one uses a Fubini type interchange of summation, which is allowed since we have summable dominants. An example in Gabor frame theory, where it becomes useful to consider this class of operators on a nonabelian group, namely a Heisenberg group with compact centre, is given in [12]. An example relating to mobile communication can be found in [6]. In this note we continue the search for more general groups, where classes of those operators are preserved under inversion.

Let $G$ be a locally compact group. A bounded operator $T$ on $L^{2}(G)$ is called convolution dominated, if it is dominated by left convolution with some $L^{1}$ function; that is, there is $f \in L^{1}(G)$ such that $|T g|(x) \leq f *|g|(x)$ almost everywhere for all $g \in L^{2}(G)$.

The set $C D(G)$ of all convolution dominated operators on $L^{2}(G)$ is a *-subalgebra of the *-algebra of all bounded operators $B\left(L^{2}(G)\right)$. In such a situation, an algebra $\mathcal{B}$ and a subalgebra $\mathcal{A} \subset \mathcal{B}$ with common unit, the question of inverseclosedness of $\mathcal{A}$ in $\mathcal{B}$ is of importance; that is, whether an element of $\mathcal{A}$ which is invertible in $\mathcal{B}$ must be invertible in $\mathcal{A}$, too. Probably the first result on inverseclosedness is due to N. Wiener [23] and widely known as Wiener's Lemma:

If a function on the unit circle with absolutely summable Fourier series has an inverse with respect to pointwise multiplication in the Banach algebra of continuous functions, then this inverse has an absolutely summable Fourier series, too.

Using results of Bochner and Phillips [5] on operator valued Fourier series, quite a few authors studied the inverse-closedness of $C D(G)$ in $B\left(L^{2}(G)\right)$ for abelian discrete groups $G[10,1,3,2,22,7]$. Using techniques from noncommutative harmonic analysis $[13,18,19,21]$, in [7] we together with K. Gröchenig treated the case of rigidly symmetric, amenable, not necessarily abelian, discrete groups (which in particular includes all nilpotent discrete groups).

In the case of nondiscrete $G$ (here the question is about $1+T$ in place of $T$, since $C D(G)$ has no identity) a measurability problem arises [8]; see also [4]. A path avoiding this is to restrict the question to the algebra $C D_{\text {reg }}(G)$ of convolution dominated operators with more regular side diagonals [8, 4]. In this note, in order to avoid this restriction, we adopt a different approach combining methods of $[15,16,17]$ with noncommutative harmonic analysis. With similar methods Farrell and Strohmer [6] looked at the generalised Heisenberg groups.

We extend the positive results to the following two classes of groups.

(1) There is a (measurable) relatively compact neighbourhood $U$ of the identity and a rigidly symmetric and amenable discrete subgroup $H \subset G$ with $h U h^{-1}=U$ for all $h \in H$ such that $\{h U\}_{h \in H}$ is a partition of $G$. 
(2) The topological commutator subgroup of $G$ is compact; that is, $G$ is a compact extension of an abelian group. If $G$ is connected, this is equivalent to saying that $G$ is an IN group [14].

Note that (1) covers nilpotent Lie groups that admit a rational structure. The real "ax $+\mathrm{b}$ " group (for this group the convolution dominated operators are not inverse closed in $\left.B\left(L^{2}(G)\right)[9]\right)$ shows that the compactness condition in (2) is needed. Conditions (1) and (2) cover all known examples where $C D(G)$ is inverse-closed in $B\left(L^{2}(G)\right.$ ). We note that groups satisfying property (2) are amenable [11, Theorem 1.2.6]. This is not so obvious in case (1). In an appendix we show amenability of such groups by establishing Følner's condition.

\section{Preliminaries}

Let $G$ be a locally compact group, $\mathcal{K}(G)$ the space of complex valued functions on $G$ with compact support, and $d x$ a left Haar measure on $G$. For a complexvalued function $f$ we denote by $\bar{f}$ its complex conjugate. For a subset $V \subset G$ we denote its closure by $\bar{V}$ and its Haar measure (provided $V$ is measurable) by $|V|$.

Let $U$ be a (measurable) relatively compact neighbourhood of the identity $e$. The following Lemma is well known.

Lemma 2.1. If $H \subset G$ satisfies $x U \cap y U=\emptyset$ for all $x \neq y$ in $H$, then, for $z \in G$ and relatively compact $K, L \subset G$, the number of all $h \in H$ with $h L \cap z K \neq \emptyset$ is dominated by $\frac{\left|\overline{K L^{-1} U}\right|}{|U|}$.

Proof. If $h L$ meets $z K$, we have $h \in z K L^{-1}$; hence $h U \subset z K L^{-1} U$. So the number of such elements cannot exceed $\frac{\overline{\mid z K L^{-1} U} \mid}{|h U|}=\frac{\left|\overline{K L^{-1} U}\right|}{|U|}$.

Let $H \subset G$ be a discrete subset, and let $U$ be a relatively compact neighbourhood of the identity $e$ such that $\{x U\}_{x \in H}$ is a partition of $G$. With this setting, we define the amalgam space

$$
\left(L^{\infty}, l^{1}\right)=\left\{f \in L^{1}(G): \sum_{k \in H}\left\|f \cdot \chi_{k U}\right\|_{\infty}<\infty\right\} .
$$

Note that if $U$ is invariant under conjugation by elements of $H$ (this will be our standard assumption below), then $|x U|=|U x|$, so $\Delta(x)=1$ for $x \in H$, and for $x \in H$ and $u \in U$ we have $\Delta(x u)=\Delta(u) \leq \sup _{u \in U} \Delta(u)<\infty$; so $G$ is unimodular.

Proposition 2.2. Given the above assumptions on $U$ and $H$, including the invariance of $U$ under conjugation by elements of $H$, the amalgam space $\left(L^{\infty}, l^{1}\right)$ is a dense two-sided ideal in $L^{1}(G)$.

Proof.

(a) $\mathcal{K}(G)$ is dense in $L^{1}(G)$, and $\mathcal{K}(G) \subset\left(L^{\infty}, l^{1}\right)$, since for $f \in \mathcal{K}(G)$ the number of $h \in H$ with $h U \cap \operatorname{supp}(f) \neq \emptyset$ is at most $\frac{\left|\overline{\operatorname{supp}(f) U^{-1} U}\right|}{|U|}$.

(b) Since in $L^{1}$ we have $g * f=\sum_{x, y \in H} g \chi_{x U} * f \chi_{y U}$, to show that $\left(L^{\infty}, l^{1}\right)$ is a left ideal in $L^{1}(G)$ it suffices to show that, for $f \in\left(L^{\infty}, l^{1}\right), g \in L^{1}(G)$, and 
$x, y \in H$, one has $g \chi_{x U} * f \chi_{y U} \in\left(L^{\infty}, l^{1}\right)$ with $\left\|g \chi_{x U} * f \chi_{y U}\right\|_{\left(L^{\infty}, l^{1}\right)} \leq$ const $\left\|g \chi_{x U}\right\|_{1}\left\|f \chi_{y U}\right\|_{\infty}$.

Now, $\left\|g \chi_{x U} * f \chi_{y U}\right\|_{\infty} \leq\left\|g \chi_{x U}\right\|_{1}\left\|f \chi_{y U}\right\|_{\infty}$ and $\operatorname{supp}\left(g \chi_{x U} * f \chi_{y U}\right) \subset$ $x \bar{U} y \bar{U}=x y \bar{U}^{2}$. The number of all $h \in H$ with $h U \cap x y \bar{U}^{2} \neq \emptyset$ is at most $\frac{\left|\bar{U}^{2} \overline{U^{-1} U}\right|}{|\bar{U}|}=: c$ by Lemma 2.1 (taking $K=y \overline{U^{2}}$ and $L=\bar{U}$ there). So

$$
\left\|g \chi_{x U} * f \chi_{y U}\right\|_{\left(L^{\infty}, l^{1}\right)} \leq c\left\|g \chi_{x U}\right\|_{1}\left\|f \chi_{y U}\right\|_{\infty} .
$$

(c) By the assumptions on $U$ and $H$, the group $G$ is unimodular; so an argument like the above shows that $\left(L^{\infty}, l^{1}\right)$ is a right ideal in $L^{1}(G)$, too.

Definition 2.3. An operator $T \in B\left(L^{2}(G)\right)$ is called convolution dominated, if there is $f \in L^{1}(G)$ dominating $T$ in the sense that

$$
|T g|(x) \leq f *|g|(x) \quad \text { a.e. } \forall g \in L^{2}(G) .
$$

Such an $f$ is automatically non-negative.

We denote the algebra of convolution dominated operators by $C D(G)$ or simply $C D$. It is normed by

$$
\|T\|_{C D}=\inf \left\{\|f\|_{L^{1}(G)}:|T g|(x) \leq f *|g|(x) \text { a.e. } \forall g \in L^{2}(G)\right\},
$$

where $T \in C D$. We denote by $C D_{\infty}$ the space of all convolution dominated operators $T$ on $L^{2}(G)$ which are dominated by convolution with some $f \in\left(L^{\infty}, l^{1}\right)$. The norm of $T \in C D_{\infty}$ is defined by

$$
\|T\|_{C D_{\infty}}=\inf \left\{\|f\|_{\left(L^{\infty}, l^{1}\right)}:|T g|(x) \leq f *|g|(x) \text { a.e. } \forall g \in L^{2}(G)\right\} .
$$

From [9, Proposition 2.3], we know that any convolution dominated operator is an integral operator with respect to a kernel. Calling kernels equivalent if they coincide locally almost everywhere (l.a.e.) on $G \times G$, we have a linear bijection between the convolution dominated operators and the equivalence classes of kernels satisfying (2.3). A kernel $t$ of such an operator $T$ satisfies

$$
T(g)(x)=\int_{G} t(x, y) g(y) d y \text {, l.a.e., } \forall g \in L^{2}(G)
$$

and

$$
|t(x, y)| \leq f\left(x y^{-1}\right) \quad \text { l.a.e. for some } f \in L^{1}(G)
$$

At the level of kernels the composition of convolution dominated operators $S$ and $T$ with respective kernels $s$ and $t$ is given by convolution of kernels

$$
s * t(x, y)=\int_{G} s(x, z) t(z, y) d z \quad \text { l.a.e.. }
$$

This formula makes sense because $S$ and $T$ are dominated by convolution with integrable functions.

In case that the operator $T$ is in $C D_{\infty}$, we can take the dominating $f$ in $\left(L^{\infty}, l^{1}\right)$. The argument in [9, Remark 2.4] shows that the infimum in (2.1) is attained. Actually the function $\sum_{i \in H} \operatorname{ess} \sup _{x y^{-1} \in i U}|t(x, y)| \cdot \chi_{i U}$ does the job. 
Remark 2.4. If $t$ is a kernel for $T$ with $|t(x, y)| \leq f\left(x y^{-1}\right)$ locally almost everywhere for some $f \in L^{1}(G)$, then $N:=\left\{(x, y)|| t(x, y) \mid>f\left(x y^{-1}\right)\right\}$ is a local null set; so $t^{\prime}:=t \chi_{U \times U \backslash N}$ is equivalent to $t$ and hence defines the same operator, and $t^{\prime}(x, y) \leq f\left(x y^{-1}\right)$ everywhere. So, replacing $t$ by $t^{\prime}$, we may replace "l.a.e." by "a.e." in (2.2) and (2.3).

Proposition 2.5. $C D_{\infty}$ is a dense ideal in $C D$.

Proof.

(a) Let $T \in C D$ be an operator with convolution kernel $k$, and let $f \in L^{1}(G)$ be given, where $|k(x, y)| \leq f\left(x y^{-1}\right)$ locally almost everywhere. For $\varepsilon>0$, there is some $0 \leq g \in \mathcal{K}(G)$ with $\|f-g\|_{1}<\varepsilon$. Let $k_{\infty}:=\operatorname{sign} k$. $(|k| \wedge M(g))$, where $M(g)(x, y):=g\left(x y^{-1}\right)$. Then $\left|k-k_{\infty}\right| \leq M(|f-g|)$. So, if $T_{\infty}$ is defined by $k_{\infty}$, we have $T_{\infty} \in C D_{\infty}$ and $\left\|T-T_{\infty}\right\|_{C D} \leq$ $\|f-g\|_{1}<\varepsilon$.

(b) For $T \in C D$ and $S \in C D_{\infty}$ with convolution kernels $t$ and $s$, respectively, there are $f \in L^{1}(G)$ and $g \in\left(L^{\infty}, l^{1}\right)$ with $|t| \leq M(f)$ and $|s| \leq M(g)$ locally almost everywhere. So

$$
|t * s(x, y)|=\left|\int_{G} t(x, z) s(z, y) d z\right| \leq \int_{G} f\left(x z^{-1}\right) g\left(z y^{-1}\right) d z=f * g\left(x y^{-1}\right) .
$$

So $T S$ is dominated by convolution with $f * g \in\left(L^{\infty}, l^{1}\right)$; hence $T S \in$ $C D_{\infty}$

(c) Analogously we see that $C D_{\infty}$ is a right ideal in $C D$.

Proposition 2.6. If $G$ is nondiscrete, then $C D_{\infty}$ has no identity.

Proof. Suppose that $G$ is nondiscrete and that $E$ is the identity of $C D_{\infty}$ with corresponding kernel $e$. If $\mathcal{V}$ is the downward directed system of compact neighbourhoods of the group identity and $e_{V}:=\frac{1}{|V|} \chi_{V}$ for $V \in \mathcal{V}$, then $\left\{e_{V}\right\}_{V \in \mathcal{V}}$ is an approximate identity of $L^{1}(G)$. Since $E \in B\left(L^{2}(G)\right)$, we have $\left\|E\left(e_{V} * g\right)-E g\right\|_{2} \rightarrow$ 0 for $g \in \mathcal{K}(G)$. Denoting $E_{V}$ the operator belonging to the kernel $(x, y) \mapsto$ $e_{V}\left(x y^{-1}\right)$, using Fubini one has $E\left(e_{V} * g\right)=\left(E E_{V}\right) g=E_{V} g$; hence

$$
\int_{G} \int_{G} e_{V}\left(x y^{-1}\right) g(y) f(x) d y d x \rightarrow \int_{G} \int_{G} e(x, y) g(y) f(x) d y d x \text { for } f \in \mathcal{K}(G) .
$$

If $f \otimes g$ is such that its support does not meet the diagonal $\left\{\left(x, x^{-1}\right) \mid x \in G\right\}$, then the left hand side vanishes for sufficiently small $V$; so the right hand side is 0 . This implies that $e=0$ locally almost everywhere outside the diagonal. Since $G$ is nondiscrete, the diagonal is a local null set; so $e=0$ locally almost everywhere; hence $E=0$ which contradicts $E\left(C D_{\infty}\right)=C D_{\infty} \neq\{0\}$.

Remark 2.7. So $C D_{\infty}(G)$ has an identity if and only if $G$ is discrete.

Since $C D_{\infty}(G)$ is a dense ideal in $C D(G)$, Remark 2.7 holds true for $C D(G)$, too.

For $z \in \mathbb{C}$, let $\operatorname{sign} z=\frac{z}{|z|}$ if $z \neq 0$, resp. 0 if $z=0$, and extend this pointwise to complex valued functions. 


\section{MATRICES OF OPERATORS AND KERNELS}

Now we shall decompose $A \in C D_{\infty}$ and its convolution kernel $a=(a(x, y))_{x, y \in G}$ as a matrix of blocks. Since $L^{2}(G)=\oplus_{i \in H} L^{2}(i U)$ (orthogonal sum of Hilbert spaces), we may divide $A$ into blocks $A_{i j}$, where $A_{i j} \in B\left(L^{2}(j U), L^{2}(i U)\right)$ is the restriction of $A$ to $L^{2}(j U)$ composed with the orthogonal projection onto $L^{2}(i U)$. If we order the finite subsets of $H \times H$ by inclusion, we have $A=\left(A_{i j}\right)_{i, j \in H}$ in the sense that the finite submatrices of $\left(A_{i j}\right)_{i, j \in H}$, when interpreted as operators on $L^{2}(G)$, converge to $A$ in the strong operator topology. If $B=\left(B_{i j}\right)_{i, j \in H}$, then $A B=\left((A B)_{i, j}\right)$ where $(A B)_{i, j}=\sum_{k \in H} A_{i k} B_{k j}$, since multiplication on bounded sets of operators is strongly continuous. So the map $A \mapsto\left(A_{i j}\right)$ is an algebra isomorphism from $C D_{\infty}$ onto its image. The map for the corresponding kernels reads $a \mapsto\left(a_{i j}\right)$ where $a_{i j}$ is the restriction of $a$ to $i U \times j U$; that is, $a_{i j}=(a(x, y))_{(x, y) \in i U \times j U}$. Note that $\left\|A_{i j}\right\|_{o p} \leq|U|\left\|a_{i j}\right\|_{\infty}$, where the infinity norm is taken on $i U \times j U$ with respect to product Haar measure. Since for $A, B \in C D_{\infty}$ the respective kernels $a$ and $b$ are dominated by $L^{1}$-functions, the convolution $a * b$ is the kernel corresponding to $A B$. (This is done with a Fubini argument, which is not valid for general kernels.) Denoting $\lambda$ the left regular representation of $G$ on $L^{2}(G)$ and $\lambda_{i}=\lambda(i), i \in H$, we define a Hilbert space isomorphism $S: \oplus_{i \in H} L^{2}(U) \rightarrow \oplus_{i \in H} L^{2}(i U)$ by $S\left(\left(u_{i}\right)_{i \in H}\right)=\left(\lambda_{i} u_{i}\right)_{i \in H}$. Then $A_{i j}^{\circ}:=\lambda_{i}^{-1} A_{i j} \lambda_{j} \in B\left(L^{2}(U)\right)$ and $\left\|A_{i j}^{\circ}\right\|_{o p}=\left\|A_{i j}\right\|_{o p}$. We have $A_{i k}^{\circ} A_{k j}^{\circ}=$ $\lambda_{i}^{-1} A_{i k} \lambda_{k} \lambda_{k}^{-1} A_{k j} \lambda_{j}=\lambda_{i}^{-1} A_{i k} A_{k j} \lambda_{j}=\left(A_{i k} A_{k j}\right)^{\circ}$. For the kernels this reads $\left(a_{i k} *\right.$ $\left.a_{k j}\right)^{\circ}=a_{i k}^{\circ} * a_{k j}^{\circ}$ and $a_{i j}^{\circ}=(a(i \xi, j \eta))_{\xi, \eta \in U}$. Thus multiplication of blocks is carried into operator composition in $B\left(L^{2}(U)\right)$, respectively convolution of kernels on $U \times$ $U$. Altogether we obtain that the map $A \mapsto\left(a_{i j}^{\circ}\right)_{i, j \in H}$ is an algebra isomorphism onto its image in the kernel-valued matrices with matrix multiplication, where the multiplication of entries is convolution of kernels on $U \times U$. If we define the involution $\left(a_{i j}^{\circ}\right)_{i, j \in H}^{*}=\left(a_{j i}^{\circ *}\right)_{i, j \in H}$, where $b^{*}(x, y)=\overline{b(y, x)}$ for any kernel $b$ on $U \times U$ and $x, y \in U$, then the map $A \mapsto\left(a_{i j}^{\circ}\right)_{i, j \in H}$ preserves the involution, too.

Remark 3.1. The reader will have noticed that, if we allow ourselves to identify the isomorphic Hilbert spaces $L^{2}(G)$ and $\oplus_{i \in H} L^{2}(U)$ and interpret the matrix $\left(a_{i j}^{\circ}\right)$ as an operator (in the canonical way), then $A \mapsto\left(a_{i j}^{\circ}\right)$ is the identity map; that is, the operator defined by $\left(a_{i j}^{\circ}\right)$ is the original $A$ again.

For $G, H$, and $U$ as above, we define different kinds of diagonals on $G \times G$.

Definition 3.2. For $k \in H$, we call $\left\{(x, y) \in G \times G \mid x y^{-1} \in k U\right\}$ the band diagonal determined by $k$, the set $\cup_{i j^{-1} \in k U}(i U \times j U)$ the approximate block diagonal determined by $k$, and $\cup_{i j^{-1}=k}(i U \times j U)$ the block diagonal determined by $k$.

Lemma 3.3. There is $n \in \mathbb{N}$ such that each approximate block diagonal meets at most $n$ band diagonals, and conversely each band diagonal meets at most $n$ approximate block diagonals (and hence can be covered by these).

Proof. Let $x, y \in G$. There are $s, t \in H$ and $\xi, \eta \in U$ with $x=s \xi$ and $y=t \eta$. If $(x, y)$ is in the band diagonal determined by $k \in H$, this means $x y^{-1} \in k U$; that is, $s \xi \eta^{-1} t^{-1} \in k U$ or $s t^{-1} \in k U^{2} U^{-1}$. If $(x, y)$ is in the approximate block 
diagonal determined by $l \in H$, this means $s t^{-1} \in l U$. So if this approximate block diagonal meets the above band diagonal, this means $l \in k U^{2} U^{-2}$ or $l U^{2} \cap k U^{2} \neq \emptyset$. For fixed $l$ (resp. $k$ ), the number of possible such $k$ (resp. $l$ ) is dominated by $\frac{\left|\overline{U^{2} U^{-2} U}\right|}{|U|}$ by Lemma 2.1 .

Corollary 3.4. $C D_{\infty}$ is bicontinuously $*$-isomorphic to the algebra of kernel valued matrices $C D_{H}:=\left\{\left(a_{i k}^{\circ}\right)_{i, k \in H}\right\}$ with norm

$\left\|\left(a_{i k}^{\circ}\right)_{i, k \in H}\right\|:=\sum_{l \in H} \sup _{i k^{-1} \in U U}\left\|a_{i k}^{\circ}\right\|_{\infty}$.

Proof. As seen before Remark 3.1, the map $A \mapsto\left(a_{i j}^{\circ}\right)_{i, j \in H}$ carries the algebra $C D_{\infty}$ isomorphically onto its image in the kernel valued matrices. Lemma 3.3 shows that this image is precisely $C D_{H}$ and that there are norm estimates both ways for this isomorphism.

Remark 3.5. If $H$ is a subgroup, then approximate block diagonals are block diagonals.

Proof. If $i, j, k \in H$ with $i j^{-1} \in k U$, then $k$ is the only element of $H$ in $k U$, since $l U \cap l^{\prime} U=\emptyset$ for $l \neq l^{\prime}$ in $H$. It follows that $i j^{-1}=k$.

\section{Spectrality of $C D_{\infty}$ And $C D_{H}$.}

Now we assume that $H$ is a (discrete) rigidly symmetric and amenable subgroup of $G$ and that the Haar measure of $G$ is normalised, so that $|U|=1$. Let $\mathcal{A}=$ $l^{\infty}\left(H, L^{\infty}(U \times U)\right)$ denote the space of all bounded functions $f: H \rightarrow L^{\infty}(U \times U)$ with pointwise linear operations, multiplication $(f, g) \mapsto f g$, where $f g(h)=f(h) *$ $g(h)$ (where $*$ denotes the convolution of kernels) and involution $f \mapsto f^{*}$, where $f^{*}(h)(u, v)=\overline{f(h)(v, u)}$, endowed with the norm $\|f\|_{\mathcal{A}}=\sup _{h \in H}\|f(h)\|_{\infty}$. Then $\mathcal{A}$ is a Banach $*$-algebra.

We denote the left regular representation of $H$ on $\mathcal{A}$ by $T$. So $\left(T_{k} f\right)(h)=$ $f\left(k^{-1} h\right)$ for $f \in \mathcal{A}$ and $h, k \in H$. The twisted $L^{1}$ algebra $\mathcal{L}=l^{1}(H, \mathcal{A}, T)$ in the sense of Leptin is the Banach space of all functions $F: H \rightarrow \mathcal{A}$ with product $F \star G(h)=\sum_{y \in H} T_{y} F(h y) G\left(y^{-1}\right)$, involution $F \mapsto F^{*}$, where $F^{*}(h)=$ $T_{h}^{-1} F\left(h^{-1}\right)^{*}$, and norm $\|F\|=\sum_{h \in H}\|F(h)\|_{\mathcal{A} \text {. }}$

Theorem 4.1. The Banach $*$-algebra $C D_{H}$ is isometrically $*$-isomorphic to $\mathcal{L}=$ $l^{1}\left(H, l^{\infty}\left(H, L^{\infty}(U \times U)\right), T\right)$. As a result $C D_{\infty}$ is bicontinuously $*$-isomorphic to $\mathcal{L}$.

Proof. Like in [7] we define a representation $R$ of $\mathcal{L}$, but this time on $L^{2}(G)=$ $\bigoplus_{i \in H} L^{2}(U)$. The image of $R$ will turn out to be $C D_{H}$. If $\delta_{h}^{m}$ denotes the $\mathcal{A}$-valued Dirac function which takes the value $m \in l^{\infty}\left(H, L^{\infty}(U \times U)\right)$ at $h$ and vanishes on $H \backslash\{h\}$, we set $R \delta_{h}^{m}=\lambda_{h} \circ M_{m}$, where $M_{m}$ is the multiplication operator $\left(\xi_{i}\right)_{i \in H} \mapsto\left(m(i) * \xi_{i}\right)_{i \in H}$, where of course $\left(\xi_{i}\right)_{i \in H} \in \bigoplus_{i \in H} L^{2}(U)=L^{2}(G)$. Then $R \delta_{h}^{m}$ coincides with the operator $T$ given by the matrix $\left(t_{i j}\right) \in C D_{H}$ with zero entries outside the $h$ diagonal $i j^{-1}=h$ and $t_{h j, j}=m(j)$ for $j \in H$. To see this, it suffices to apply both operators to $\left(\delta_{i, k} \xi\right)_{i \in H}$, where $\xi \in L^{2}(U)$ and $k \in H$. We have $T\left(\delta_{i, k} \xi\right)=\left(\eta_{i}\right)$, where $\eta_{i}=\delta_{i, h k} m(k) * \xi$, which equals $R \delta_{h}^{m}\left(\delta_{i, k} \xi\right)$ (note that $\lambda_{h}$ permutes the $L^{2}(U)$-blocks of $L^{2}(G)$, shifting the $k$ block to the $h k$ block). 
Clearly $\left\|\delta_{h}^{m}\right\|_{\mathcal{L}}=\|T\|_{C D_{H}}=\|m\|_{\infty}$. Extending $R$ by linearity and continuity we obtain an isometric linear isomorphism from $\mathcal{L}$ onto $C D_{H}$. Consider $\delta_{h}^{m}$ and $\delta_{k}^{n}$ with $h, k \in H$ and $m, n \in \mathcal{A}=l^{\infty}\left(H, L^{\infty}(U \times U)\right)$.

$$
\begin{aligned}
R \delta_{h}^{m} R \delta_{k}^{n} & =\lambda_{h} M_{m} \lambda_{k} M_{n} \\
& =\lambda_{h k} \lambda_{k}^{-1} M_{m} \lambda_{k} M_{n}=\lambda_{h k} M_{\left(T_{k}^{-1} m\right) n} \\
& =R \delta_{h k}^{\left(T_{k}^{-1} m\right) n}=R\left(\delta_{h}^{m} * \delta_{k}^{n}\right) .
\end{aligned}
$$

This implies that $R$ is multiplicative. Finally

$$
\begin{aligned}
\left(R \delta_{h}^{m}\right)^{*} & =\left(\lambda_{h} M_{m}\right)^{*}=M_{m^{*}} \lambda_{h}^{-1} \\
& =\lambda_{h}^{-1} M_{T_{h} m^{*}}=R\left(\delta_{h^{-1}}^{T_{h} m^{*}}\right) ;=R\left(\left(\delta_{h}^{m}\right)^{*}\right) .
\end{aligned}
$$

So $R$ is a $*$-isomorphism from $\mathcal{L}=l^{1}\left(H, l^{\infty}\left(H, L^{\infty}(U \times U)\right)\right)$ onto $C D_{H}$ (resp. $C D_{\infty}$ ), which is isometric (resp. bicontinuous). At the same time $R$ is a $*-$ representation of $\mathcal{L}$ on $L^{2}(G)$.

Lemma 4.2. Let $K_{2, \infty}$ be the space of (equivalence classes of) measurable kernels on $U \times U$ with

$$
\|k\|_{2, \infty}=\operatorname{ess} \sup _{y \in U}\|k(\cdot, y)\|_{2}<\infty .
$$

Then $L^{\infty}(U \times U)$ is a right Banach $K_{2, \infty}$ module, and $K_{2, \infty}$ is a left $L^{2}(U \times U)$ module for the convolution of kernels.

Proof. In order to avoid additional constants coming up, we normalise Haar measure on $G$ so that $|U|=1$.

(a) Let $g \in L^{\infty}(U \times U)$ and $k \in K_{2, \infty}$. Since

$$
\begin{aligned}
|g * k(r, s)| & =\left|\int_{U} g(r, t) k(t, s) d s\right| \leq\|g(r .)\|_{\infty}\|k(\cdot, s)\|_{1} \\
& \leq\|g\|_{\infty}\|k(\cdot, s)\|_{2} \leq\|g\|_{\infty}\|k\|_{2, \infty} \quad \text { a.e. }
\end{aligned}
$$

we have

$$
\|g * k\|_{\infty} \leq\|g\|_{\infty}\|k\|_{2, \infty}
$$

(b) Let $h \in L^{2}(U \times U)$ and $k \in K_{2, \infty}$. Then

$$
|h * k(r, s)| \leq\|h(r, \cdot)\|_{2}\|k(\cdot, s)\|_{2} \leq\|h(r, \cdot)\|_{2}\|k\|_{\infty, 2} \quad \text { a.e. ; }
$$

SO

$$
\|h * k(\cdot, s)\|_{2}^{2} \leq \int_{U} \int_{U}|h(r, u)|^{2} d u d r\|k\|_{2, \infty}^{2} \leq\|h\|_{2}^{2}\|k\|_{2, \infty}^{2} .
$$

Hence

$$
\|h * k\|_{2, \infty} \leq\|h\|_{2}\|k\|_{2, \infty}
$$

Definition 4.3. A subalgebra $\mathcal{A}$ of an algebra $\mathcal{B}$ is called a spectral subalgebra of $\mathcal{B}$ or spectral in $\mathcal{B}$, if for every $a \in \mathcal{A}$ the spectrum of $a$ in $\mathcal{A}$ coincides with its spectrum in $\mathcal{B}$ except perhaps for the value zero. (Without the removal of zero the notion would be equivalent to inverse-closedness). 
In the following remark for an element $a$ of a Banach algebra $\mathcal{A}$ we denote its spectral radius by $r(a)$.

Remark 4.4 .

(a) $L^{\infty}(U \times U)$ and the Hilbert-Schmidt kernels $L^{2}(U \times U)$ are Banach algebras for convolution, and so is $K_{2, \infty}$, since \|\|$_{2, \infty} \geq\|\|_{2}$ on $K_{2, \infty}$.

(b) Since in general one sided ideals are spectral subalgebras, $\left(L^{\infty}(U \times U), *\right)$ is spectral in $K_{2, \infty}$ which is spectral in the algebra of Hilbert-Schmidt operators on $L^{2}(U)$, which in turn is spectral in $B\left(L^{2}(U)\right)$. So $\left(L^{\infty}(U \times U), *\right)$ is spectral in $B\left(L^{2}(U)\right)$.

(c) The closure of $L^{\infty}(U \times U)$ in $B\left(L^{2}(U)\right)$ is its $C^{*}$-hull $C^{*}\left(L^{\infty}(U \times U)\right)$. For $a \in L^{\infty}(U \times U)$ and the operator $A$ defined by it, one has $\|A\|=$ $r\left(A^{*} A\right)^{\frac{1}{2}}=r\left(a^{*} a\right)^{\frac{1}{2}}$ by $(\mathrm{b})$. Since $\|\pi(a)\| \leq r\left(a^{*} a\right)^{\frac{1}{2}}$ for any Hilbert space *-representation of $L^{\infty}(U \times U)$, the norm $a \mapsto\|A\|$ is the greatest $C^{*}$ seminorm on it. Hence the $C^{*}$-hull of $L^{\infty}(U \times U)$ is its closure in $B\left(L^{2}(U)\right)$.

(d) The argument in (c) shows that if $\mathcal{A}$ is a Banach *-algebra contained in a $C^{*}$-algebra $\mathcal{B}$ and $\mathcal{A}$ is spectral in $\mathcal{B}$, then the closure of $\mathcal{A}$ in $\mathcal{B}$ is the $C^{*}$ hull $C^{*}(\mathcal{A})$. (Without the spectrality assumption this is false. Consider $L^{1}(G) \subset B\left(L^{2}(G)\right)$ for a nonamenable group $G$.)

The set $\mathcal{C}=l^{\infty}\left(H, B\left(L^{2}(U)\right)\right.$ with pointwise operations and involution (where multiplication in $B\left(L^{2}(U)\right)$ is composition) is a $C^{*}$-algebra. The left regular representation of $H$ on $\mathcal{C}$ is denoted by $T$. So $T_{k} f(h)=f\left(k^{-1} h\right)$ for $f$ in $\mathcal{C}$ and $h, k \in H$. In analogy to the beginning of this section we consider the twisted $L^{1}$-algebra $\mathcal{B}=l^{1}\left(H, l^{\infty}\left(H, B\left(L^{2}(U)\right), T\right)\right.$ and define the $*$-representation $R$ of $\mathcal{B}$ like for $\mathcal{L}$ in the proof of Theorem 4.1 .

\section{Proposition 4.5.}

(a) The closure of $R(\mathcal{B})$ in $B\left(L^{2}(G)\right)$ is its $C^{*}$-hull $C^{*}(\mathcal{B})$.

(b) $R(\mathcal{B})$ is spectral in $B\left(L^{2}(G)\right)$.

(c) $C D_{\infty}(G)$ is spectral in $B\left(L^{2}(G)\right)$. In particular, $C D_{\infty}(G)$ is a symmetric Banach *-algebra.

Proof. Like in the proof of Theorem 4.1, we let

$$
M: l^{\infty}\left(H, B\left(L^{2}(U)\right)\right) \rightarrow B\left(l^{2}\left(H, L^{2}(U)\right)\right)=B\left(L^{2}(G)\right)
$$

be the $*$-representation $c \mapsto M_{c}$, where $M_{c}\left(\xi_{i}\right)_{i \in H}=\left(c(i) \xi_{i}\right)_{i \in H}$ for $\left(\xi_{i}\right)_{i \in H} \in$ $l^{2}\left(H, L^{2}(U)\right)$. Since $H$ is amenable and $l^{\infty}\left(H, B\left(L^{2}(U)\right)\right)$ is a $C^{*}$ algebra, by Leptin [20] the $M$-regular representation is a maximal representation of $\mathcal{B}$. It is weakly equivalent to $R$.

To see this one slightly modifies the proof of [7, Prop 3]. Since $R$ happens on $L^{2}(G)=l^{2}\left(H, L^{2}(U)\right)$ and $\lambda^{M}$ happens on $l^{2}\left(H, L^{2}(G)\right)=l^{2}\left(H \times H, L^{2}(U)\right)$, we 
work on this last space, which we denote $\mathcal{H}$ for short. Like in [7], let $R^{\omega}$ denote the extension of $R$ from $L^{2}(G)$ to $\mathcal{H}$ defined by letting the operator $R(f)=$ $\sum_{y \in H} \lambda(y) \circ M_{f(y)}$, where $f \in \mathcal{L}$, act on the first coordinate only. That is,

$$
R^{\omega}(f) \xi(x, z)=\sum_{y \in H} f(y)\left(y^{-1} x\right) \xi\left(y^{-1} x, z\right) \quad \text { for } \xi \in \mathcal{H}, x, z \in H .
$$

The operator $S: \mathcal{H} \rightarrow \mathcal{H}$ defined by $S \xi(x, z)=\xi(x z, z)$ is unitary and intertwines $R^{\omega}$ and $\lambda^{M}$ as in [7].

So $R^{\omega}$ and in turn $R$ are maximal representations of $\mathcal{L}$.. Thus the closure of $R(\mathcal{B})$ in $B\left(L^{2}(G)\right)$ is $C^{*}(\mathcal{B})$. This proves (a).

For $f \in \mathcal{B}$ the operator $R(f)$ can be viewed as an $l^{1}$-sum of its diagonals. Each diagonal (with zero entries outside the diagonal) is a bounded operator on $L^{2}(G)$, and its operator norm is the supremum of the $B\left(L^{2}(G)\right)$-norms of its entries. So the *-isomorphism $f \mapsto R(f)$ maps $\mathcal{B}$ isometrically into $l^{1}\left(H, B\left(L^{2}(G)\right)\right.$. In particular, $R(\mathcal{B})$ is a complete and hence closed $*$-subalgebra of $l^{1}\left(H, B\left(L^{2}(G)\right)\right)$ $\cong l^{1}(H) \hat{\otimes} B\left(L^{2}(G)\right)$. The latter is symmetric, since $H$ is rigidly symmetric. So $R(\mathcal{B})$ is symmetric, too. By [7] and (a), $R(\mathcal{B})$ is spectral in $B\left(L^{2}(G)\right)$. This proves (b).

By Lemma $4.2, \mathcal{L}$ is a right ideal in $l^{1}\left(H, l^{\infty}\left(H, K_{2, \infty}\right)\right)$, which is a left ideal in $l^{1}\left(H, l^{\infty}\left(H, L^{2}(U \times U)\right)\right)$, which is a two-sided ideal in $\mathcal{B} \cong R(\mathcal{B})$ which by (b) is spectral in $\left.B\left(L^{2}(G)\right)\right)$. So $C D_{\infty}(G) \cong R(\mathcal{L}) \cong \mathcal{L}$ is spectral in $B\left(L^{2}(G)\right)$.

Let 1 denote the identity operator in $B\left(L^{2}(G)\right)$.

Corollary 4.6. $\mathbb{C} 1+C D_{\infty}(G)$ is inverse-closed in $B\left(L^{2}(G)\right)$.

Proof. If $G$ is discrete, this is contained in [7] ( $\mathbb{C} 1$ may be omitted here, since $\left.1 \in C D_{\infty}=C D\right)$. Hence we may assume that $G$ is nondiscrete. Then $C D_{\infty}(G)$ has no identity and is spectral in $B\left(L^{2}(G)\right)$ which has the identity 1 . So the assertion follows.

Theorem 4.7. $\mathbb{C} 1+C D(G)$ is inverse-closed in $B\left(L^{2}(G)\right)$.

Proof. If $G$ is discrete, then $C D_{\infty}(G)=C D(G)$; so this case is covered by Corollary 4.6. In the non-discrete case: $\mathbb{C} 1+C D_{\infty}(G)$ is inverse-closed in $B\left(L^{2}(G)\right)$, and $C D_{\infty}(G)$ is a dense two-sided ideal in $C D(G)$. (See for instance [17] for the argument.)

\section{Groups with compact commutator}

Consider a locally compact group $G$ with compact topological commutator subgroup $C=\overline{[G, G]}$. We denote $p_{C}: G \rightarrow G / C$ the canonical projection. $G / C$ is a locally compact abelian group; so $G / C=\mathbb{R}^{d} \times J$, where $d \in \mathbb{N} \cup\{0\}$ and $J$ contains a compact open subgroup $K$. Let $R \subset J$ be a complete set of representatives of $J / K, U:=\left(p_{C}^{-1}\left(\left[-\frac{1}{2}, \frac{1}{2}\right)^{d} \times K\right)\right.$, and let $H \subset G$ be a complete set of representatives of $\mathbb{Z}^{d} \times R \subset G / C$. Then $U$ is relatively compact, measurable, and $H$-invariant. Furthermore $\{h U\}_{h \in H}$ is a partition of $G$. The set $h U$ does not depend on the choice of the representatives in $J$ and in $G$; so we may write $\dot{h} U$ for $h U$ with $\dot{h} \in D:=\mathbb{Z}^{d} \times J / K$. 
Every $h \in H$ is of the form $h=\operatorname{rep}_{C}(m, r)$, where $r e p_{C}$ denotes a representative of $(m, r) \in G / C, m \in \mathbb{Z}^{d}$, and $r$ is a representative $r e p_{K}(r K)$ of $r K \in J / K$ in $J$. So $h U=p_{C}^{-1}\left(\left(m+\left[-\frac{1}{2}, \frac{1}{2}\right)^{d}\right) \times r K\right)$ which means that $h U$ only depends on $\dot{h}:=(m, r K)=\left(i d \times p_{K}\right)\left(p_{C}(h)\right) \in D$. If $k=\operatorname{rep}_{C}\left(m^{\prime}, r^{\prime}\right)$ and $\dot{h}=\dot{k}$, it follows that $m=m^{\prime}$ and $r K=r^{\prime} K$; hence $h=k$. So the map $h \mapsto \dot{h}$ is bijective from $H$ to $D$. Note also that $(h k \dot{k})=\dot{h} \dot{k}$ and $\left(h^{-1}\right)=(\dot{h})^{-1}$ (but unlike $D$ the set $H$ need not be a group).

Proposition 5.1. The approximate block diagonals $(h U, k U)_{h k^{-1} \in l U}$ of Definition 3.2 are exactly the block diagonals $(\dot{h} U, \dot{k} U)_{\dot{h}^{-1}=i}$.

Proof.

(a) $h k^{-1} \in l U$ implies $l^{-1} h k^{-1} \in U$. The formula for $h \mapsto \dot{h}$ is meaningful on all of $G$ and defines an homomorphism onto $\mathbb{R}^{d} \times J / K$. Now $\dot{l}^{-1} \dot{h} \dot{k}^{-1} \in$ $\dot{U} \cap D=\{0\}$ (where $\dot{U}$ denotes the set $\{\dot{u}: u \in U\}$ ). So $\dot{h} \dot{k}^{-1}=\dot{l}$.

(b) If $\dot{h} \dot{k}^{-1}=\dot{l}$, then $h k^{-1}$ differs from $l$ by an element $w \in G$ with $\dot{w}=0$; that is, $w \in p_{c}^{-1}(\{0\} \times K)$. So $h k^{-1}=l w \in l U$.

Corollary 3.4 and Proposition 5.1 now imply the following theorem.

Theorem 5.2. If the (topological) commutator subgroup $\overline{[G, G]}$ is compact, then $C D_{\infty}$ is bicontinuously *-isomorphic to the *-algebra of kernel-valued matrices

$$
C D_{H}=\left\{\left(a_{h k}^{\circ}\right)_{h, k \in H}\right\}=\left\{\left(a_{\dot{h} \dot{k}}^{\circ}\right)_{\dot{h}, \dot{k} \in D}\right\},
$$

with norm

$$
\left\|\left(a_{\dot{h} \dot{k}}^{\circ}\right)_{\dot{h}, \dot{k} \in D}\right\|=\sum_{i \in D} \sup _{\dot{h} \dot{k}^{-1}=\dot{l}}\left\|a_{\dot{h} \dot{k}}^{\circ}\right\|_{\infty}
$$

the infinity norm being taken on $h U \times k U$ with product Haar measure on it.

Like in the beginning of section 3, we have $L^{2}(G)=\oplus_{h \in H} L^{2}(h U)=\oplus_{h \in H} L^{2}(U)$ with canonical isomorphisms, and as we just have seen we may replace the labelling $h \in H$ by $\dot{h} \in \dot{H}=D$. Although $D=\mathbb{Z}^{d} \times J / K$ is not a subgroup of $G$; in general, it acts on $L^{2}(G)=\oplus_{h \in D} L^{2}(U)$ by permuting the $L^{2}(U)$ blocks. Denoting the action of $i \in D$ by $\lambda_{i}$, we have $\lambda_{i}\left(f_{\dot{h}}\right)_{\dot{h} \in D}=\left(f_{i^{-1} \dot{h}}\right)_{\dot{h} \in D}$ for $\left(f_{\dot{h}}\right)_{\dot{h} \in D} \in \oplus_{\dot{h} \in D} L^{2}(U)$. At the level of $L^{2}(G)=\oplus_{\dot{h} \in H} L^{2}(h U)$ we would have $\lambda_{i} g_{h}=\lambda_{r(i \hbar h)} \lambda_{h^{-1}} g_{h}=\lambda_{r(i h) h^{-1}} g_{h}$, for $g_{h} \in L^{2}(h U)$, where $r(i \dot{h})$ denotes the unique $k \in H$ with $\dot{k}=i \dot{h}$. This is a consequence of respecting our identifications. Note that $\lambda_{i}$ may be different from $\lambda_{l}$.

We also define the action $T$ of $D=\dot{H}$ on $\mathcal{A}=l^{\infty}\left(H, L^{\infty}(U \times U)\right)=$ $l^{\infty}\left(D, L^{\infty}(U \times U)\right)$ by $T_{\dot{k}} f(\dot{h})=f\left(\dot{k}^{-1} \dot{h}\right)$ for $f \in \mathcal{A}$ and $\dot{h}, \dot{k} \in D$. For $m \in \mathcal{A}$, the multiplication operator $M_{m}$ on $\oplus_{\dot{h} \in D} L^{2}(U \times U)$ is defined by $\left(M_{m} g\right)(\dot{h})=$ $m(\dot{h}) * g(\dot{h})$ for $\dot{h} \in D, g \in \oplus_{\dot{h} \in D} L^{2}(U)$ where $*$ means convolution of kernels in $L^{\infty}(U \times U)$. Like in the case where $H$ is a subgroup, we have $\lambda_{\dot{k}} M_{m} \lambda_{\dot{k}^{-1}}=M_{T_{\dot{k}} m}$.

Letting $\mathcal{L}=l^{1}(D, \mathcal{A}, T)$, denote the twisted $L^{1}$-algebra like in the beginning of section 4 (replace $H$ and its elements by $D$ and the respective elements); we may repeat the proof of Theorem 4.1 to obtain its analogue. 
Theorem 5.3. The Banach $*$-algebra $C D_{H}$ is isometrically $*$-isomorphic to $\mathcal{L}=$ $l^{1}\left(D, l^{\infty}\left(D, L^{\infty}(U \times U)\right), T\right)$. As a result $C D_{\infty}$ is bicontinuously $*$-isomorphic to $\mathcal{L}$.

Continuing as in section 4 with $D$ in place of $H$, we obtain the analoga of Corollary 4.6 and Theorem 4.7.

Corollary 5.4. $\mathbb{C} 1+C D_{\infty}(G)$ is inverse-closed in $B\left(L^{2}(G)\right)$.

Theorem 5.5. $\mathbb{C} 1+C D(G)$ is inverse-closed in $B\left(L^{2}(G)\right)$.

\section{ApPEndix}

Here we shall derive the amenability of a locally compact group $G$ satisfying our assumption (1).

Lemma 6.1. If $K \subset G$ is compact, then it can be covered by finitely many sets $h U$ with $h \in H$.

Proof.

(i) First note that, due to our assumptions, $H$ is not only discrete but uniformly discrete. This implies that a net $\left(h_{\mu}\right)$ in $H$ cannot converge to a point outside $H$, and if $h_{\mu} \rightarrow h \in H$, then $h_{\mu}=h$ from some index $\mu_{0}$ onwards.

(ii) Suppose that $K \subset G$ is compact and meets infinitely many sets $h_{n} U$, $h_{n} \in H, n \in \mathbb{N}$, and $h_{n} \neq h_{m}$ for $n \neq m$. For $n \in \mathbb{N}$, choose $k_{n} \in K \cap h_{n} U$; so $k_{n}=h_{n} u_{n}$ with $u_{n} \in U$. Choose a convergent subnet $\left(k_{\mu}\right)$ of $\left(k_{n}\right)_{n \in \mathbb{N}}$; $k_{\mu} \rightarrow k \in K$ say. Choose a subnet $\left(k_{\mu^{\prime}}\right)$ of $\left(k_{\mu}\right)$ such that $k_{\mu^{\prime}}=h_{\mu^{\prime}} u_{\mu^{\prime}}$ with $u_{\mu^{\prime}} \rightarrow \bar{u} \in \bar{U}$. Then $h_{\mu^{\prime}}=k_{\mu^{\prime}} u_{\mu^{\prime}}^{-1} \rightarrow k \bar{u}^{-1}$. This contradicts the fact that $\left(h_{\mu^{\prime}}\right)$, being a subnet of $\left(h_{n}\right)_{n \in \mathbb{N}}$, cannot converge (according to (i)).

Now we show that $G$ satisfies the Følner condition (see $[11,3.6]$ ). For a compact $K \subset G$, let $K$ be covered by finitely many $h_{i} U, i=1, \ldots, M$, and let the compact set $\overline{U^{2}} \cup \overline{U^{-1} U}$ be covered by $k_{j} U, j=1, \ldots, L$. Let $\epsilon>0$ be arbitrary. Since $H$ satisfies the Følner condition, for $\cup_{i, j}\left\{k_{j} h_{i}, k_{j} h_{i}^{-1}\right\}$, there exists a nontrivial finite set $E \subset H$ such that $\left|k_{j} h_{i} E \Delta E\right| \leq \frac{\varepsilon}{L}|E|$ and $\left|k_{j} h_{i}^{-1} E \Delta E\right| \leq \frac{\varepsilon}{L}|E|$ for $i=1, \ldots, M, j=1, \ldots, L$. (On the discrete group $H$ the Haar measure coincides with the counting measure, thus for $E \subset H$ the cardinality of $E$ is $|E|$.) Then for $x \in K, x=h_{i} u$ say, we have

$$
\begin{aligned}
\left|h_{i} u U E \backslash U E\right| & \leq\left|h_{i} U^{2} E \backslash U E\right|=\left|U^{2} h_{i} E \backslash U E\right| \\
& \leq\left|\bigcup_{j=1}^{L} k_{j} U h_{i} E \backslash U E\right|=|U|\left|\bigcup_{j=1}^{L} k_{j} h_{i} E \backslash E\right| \\
& \leq|U| \sum_{j=1}^{L}\left|k_{j} h_{i} E \backslash E\right| \leq|U| \varepsilon|E|=\varepsilon|U E|
\end{aligned}
$$


Similarly

$$
\begin{aligned}
\left|U E \backslash h_{i} u U E\right| & \leq\left|u^{-1} h_{i}^{-1} U E \backslash U E\right| \leq\left|U^{-1} U h_{i}^{-1} E \backslash U E\right| \\
& \leq\left|\bigcup_{j=1}^{L} k_{j} U h_{i}^{-1} E \backslash U E\right| \leq|U| \sum_{j=1}^{L}\left|k_{j} h_{i}^{-1} E \backslash E\right| \\
& \leq|U| \varepsilon|E|=\varepsilon|U E| .
\end{aligned}
$$

So we obtain, for $x=h_{i} u \in K$,

$$
\begin{aligned}
|x U E \Delta U E| & =\left|\left\{h_{i} u U E \backslash U E\right\} \cup\left\{U E \backslash h_{i} u U E\right\}\right| \\
& \leq\left|h_{i} u U E \backslash U E\right|+\left|U E \backslash h_{i} u U E\right| \leq 2 \varepsilon|U E| .
\end{aligned}
$$

Acknowledgements. We thank Karlheinz Gröchenig for his careful reading of a preliminary version of this note and Karl H. Hofmann for a discussion on the structure of the groups we consider. We thank Michael Cowling for a fruitful discussion on the amenability of these groups.

\section{REFERENCES}

1. A. G. Baskakov, Abstract harmonic analysis and asymptotic estimates for elements of inverse matrices, (Russian); translated from Mat. Zametki 52 (1992), no. 2, 17-26, 155 Math. Notes 52 (1992), no. 1-2, 764-771 (1993).

2. A. G. Baskakov, Asymptotic estimates for elements of matrices of inverse operators, and harmonic analysis, (Russian); translated from Sibirsk. Mat. Zh. 38 (1997), no. 1, 14-28, i Siberian Math. J. 38 (1997), no. 1, 10-22.

3. A. G. Baskakov, Estimates for the elements of inverse matrices, and the spectral analysis of linear operators, (Russian); translated from Izv. Ross. Akad. Nauk Ser. Mat. 61 (1997), no. 6, 3-26 Izv. Math. 61 (1997), no. 6, 1113-1135.

4. I. Beltiţă and D. Beltiţă, Erratum to: Inverse-closed algebras of integral operators on locally compact groups, Ann. Henri Poincaré 16 (2015), no. 5, 1307-1309.

5. S. Bochner and R. S. Phillips, Absolutely convergent Fourier expansions for noncommutative normed rings, Ann. of Math. (2), 43 (1942), 409-418.

6. B. Farrell and T. Strohmer, Inverse-closedness of a Banach algebra of integral operators on the Heisenberg group, J. Operator Theory 64 (2010), no. 1, 189-205.

7. G. Fendler, K. Gröchenig, and M. Leinert, Convolution-dominated operators on discrete groups, Integral Equations Operator Theory 61 (2008), no. 4, 493-509.

8. G. Fendler, K. Gröchenig, and M. Leinert, Convolution-dominated integral operators, Noncommutative harmonic analysis with applications to probability II, 121-127, Banach Center Publ., 89, Polish Acad. Sci. Inst. Math., Warsaw, 2010.

9. G. Fendler and M. Leinert, On convolution dominated operators, Integral Equations Operator Theory 86 (2016), no. 2, 209-230.

10. I. Gohberg, M. A. Kaashoek, and H. J. Woerdeman, The band method for positive and contractive extension problems, J. Operator Theory 22 (1989), no. 1, 109-155.

11. F. P. Greenleaf, Invariant means on topological groups and their applications, Van Nostrand Mathematical Studies, No. 16, Van Nostrand Reinhold Co., New York-Toronto, Ont.London, 1969.

12. K. Gröchenig and M. Leinert, Wiener's lemma for twisted convolution and Gabor frames, J. Amer. Math. Soc. 17 (2004), 1-18.

13. A. Hulanicki, On the spectrum of convolution operators on groups with polynomial growth, Invent. Math. 17 (1972), 135-142.

14. K. Iwasawa, Topological groups with invariant compact neighborhoods of the identity, Ann. of Math. (2) 54 (1951), 345-348. 
15. V. G. Kurbatov, Algebras of difference and integral operators, Funktsional. Anal. i Prilozhen. 24 (1990), no. 2, 87-88.

16. V. G. Kurbatov, Functional differential operators and equations, Mathematics and its Applications, 473. Kluwer Academic Publishers, Dordrecht, 1999.

17. V. G. Kurbatov, Some algebras of operators majorized by a convolution, International Conference on Differential and Functional Differential Equations (Moscow, 1999). Funct. Differ. Equ. 8 (2001), no. 3-4, 323-333.

18. H. Leptin, Verallgemeinerte $L^{1}$-Algebren, Math. Ann. 159 (1965), 51-76.

19. H. Leptin, Verallgemeinerte $L^{1}$-Algebren und projektive Darstellungen lokal kompakter Gruppen, I, Invent. Math. 3 (1967), 257-281.

20. H. Leptin, Darstellungen verallgemeinerter $L^{1}$-Algebren, (German) Invent. Math. 5 (1968), $192-215$.

21. H. Leptin and D. Poguntke, Symmetry and non-symmetry for locally compact groups, J. Funct. anal. 33 (1979), 119-134.

22. Q. Sun, Wiener's lemma for infinite matrices with polynomial off-diagonal decay, C. R. Math. Acad. Sci. Paris 340 (2005), no. 8, 567-570.

23. N. Wienerm, Tauberian theorems, Ann. of Math. (2) 33 (1932), no. 1, 1-100.

${ }^{1}$ Finstertal 16, D-69514 Laudenbach, Germany.

E-mail address: gero.fendler@univie.ac.at

${ }^{2}$ Institut für Angewandte Mathematik, Universität Heidelberg, Im Neuenheimer Feld 205, D-69120 Heidelberg, Germany.

E-mail address: leinert@math. uni-heidelberg.de 